\title{
Ethnobotanique et analyse phytochimique qualitative de Pteridium aquilinum (L.) Kühn (Dennstaedtiaceae), une Ptéridophyte utilisée comme plante médicinale en Côte d'Ivoire
}

\author{
Lydie Marie Dominique ADOU ${ }^{1 *}$, Mamidou Withabouna KONE ${ }^{2}$, Joseph IPOU IPOU $^{1}$ et \\ Edouard Kouakou N'GUESSAN ${ }^{1}$ \\ ${ }^{1}$ Université Félix HOUPHOUET BOIGNY, UFR Biosciences, Laboratoire de Botanique, 22 BP 582 Abidjan \\ 22, Côte d'Ivoire. \\ ${ }^{2}$ Université NANGUI ABROGOUA, UFR Sciences de la Nature Laboratoire de Botanique et Physiologie \\ Végétale, 02 BP 801 Abidjan 02, Côte d'Tvoire. \\ *Auteur correspondant, E-mail : essiganmd@gmail.com ; Tel : +22507671309
}

\section{RESUME}

En Côte d'Ivoire, les études ethnobotaniques ont longtemps été réservées aux spermaphytes. Très rarement, ces études ont concerné les Ptéridophytes. Il est donc nécessaire de connaître les différentes utilisations de ces taxons. La présente étude vise à valoriser Pteridium aquilinum, une Ptéridophyte communément appelé fougère aigle. Cette valorisation consiste à montrer les différents usages de la plante et à déterminer les différents groupes de composés chimiques contenus dans cette dernière pour tenter d'expliquer les effets thérapeutiques potentiels de la plante. La démarche méthodologique a consisté à réaliser une enquête ethnobotanique auprès des populations de la zone d'étude. Le type de méthode est une interview directe, qui consiste à interroger les populations. Leurs réponses sont transcrites sur une fiche d'enquête adaptée, conçue à cet effet. Ainsi une étude phytochimique a été réalisée avec les extraits de Pteridium aquilinum. Il ressort de ces investigations que sur 99 personnes interrogées, 29 soit $29 \%$ connaissent la plante ou en savent l'utilité. La plante entière est utilisée pour la préparation des recettes médicamenteuses dans $(66 \%)$ des cas. La préparation des recettes médicamenteuses fait intervenir majoritairement la décoction $66 \%$. On note également divers modes d'administration de ces médicaments : boisson, bain de vapeur et onguent utilisé chacun à 33\%. Les investigations phytochimiques menées sur des extraits de la plante suivant la méthode de la caractérisation en tube, ont révélé que Pteridium aquilinum renferme des stérols, des polyterpènes, des polyphénols, des flavonoïdes, des tanins catéchiques et des substances quinoniques. Ce sont ces substances qui confèrent à la plante son aptitude à traiter certaines maladies. En conclusion, Pteridium aquilinum est utilisée comme plante médicinale sous diverses formes de préparations médicamenteuses dans la lutte contre le rhumatisme et les convulsions des nouveau-nés.

(C) 2016 International Formulae Group. All rights reserved.

Mots clés: Composés chimiques, Fougère, Phytochimie, Ptéridophyte, Sud-Est de la Côte d'Ivoire. 


\title{
Ethnobotanical and qualitative phytochemical analysis of Pteridium aquilinum. (L.) Kühn (Dennstaedtiaceae), a Pteridophyte used as medicinal plant in Côte d'Ivoire
}

\begin{abstract}
In Côte d'Ivoire, ethnobotanical studies have concerned seed plants or spermatophytes for a long time. They very rarely dealt with Pteridophytes. That is why it is necessary to know the different uses of these taxa. The present study aims to increase the value of Pteridium aquilinum, a Pteridophyte commonly called bracken. This valuation is to show the different uses of the plant and determine the different chemical components it contains in order to explain its therapeutic potentials. The methodological approach was to carry out an ethnobotanical survey among people in the study area. The type of method is a live interview which involves interviewing people. Their responses were transcribed on an adapted survey sheet, designed for this purpose. A phytochemical study was also conducted with the extracts of Pteridium aquilinum. From these investigations on 99 respondents, 29 or $29 \%$ know the plant or know the utility. The whole plant is used for the preparation of drug recipes in $(66 \%)$ of cases. The preparation of medicinal recipes mostly involve decoction $(66 \%)$. There are also various modes of administration of these drugs: drink, steam bath and ointment, used each at $33 \%$. Phytochemical investigations on extracts of the plant following the method of characterization tube revealed that Pteridium aquilinum contains sterols, polyterpenes, polyphenols, flavonoids, catechin tannins and quinone substances. These are the substances that give the plant its ability to treat certain diseases. In conclusion, Pteridium aquilinum is used as a medicinal plant in various forms of pharmaceutical preparations in the fight against rheumatism and convulsions of new-borns.
\end{abstract}

(C) 2016 International Formulae Group. All rights reserved.

Key words: Chemical components, Ferns, Phytochemistry, Pteridophytes, South-East Côte d'Ivoire.

\section{INTRODUCTION}

Depuis la préhistoire, les plantes sont utilisées dans la médecine traditionnelle par l'homme. En effet, les populations rurales n'ont généralement pas accès aux produits pharmaceutiques à cause de leurs coûts élevés (Gning et al., 2007). Cette situation emmène les malades à s'orienter vers les remèdes traditionnels. L'importance des plantes médicinales est donc unanimement reconnue. En Afrique particulièrement, plusieurs études ethnobotaniques ont été menées; il s'agit entre autres, des travaux de Balde et al. (2006) en guinée; Sanogo (2006) au Mali ; Mangambu et al. (2012) en RDC; Dossou et al. (2012) au Bénin. La Côte d'Ivoire n'est pas restée en marge de cette prise de conscience. Ainsi diverses études ethnobotaniques ont étés menées par plusieurs chercheurs (Ouattara, 2006 ; Tra Bi et al., 2008 ; Azokou, 2010; N'guessan et al., 2010). Il est à remarquer que si les premières études ethnobotaniques ont été essentiellement basées sur les spermatophytes (Singh et al., 2012), les champs d'investigation sont actuellement diversifiés et portent sur tous les embranchements du règne végétal (Mangambu et al., 2012).

Cependant, la méconnaissance des composés chimiques responsables des activités biologiques des plantes utilisées peut entraîner de graves problèmes à ceux qui les utilisent. En effet, les doses employées restent imprécises (Bruneton, 2009). Divers études pharmacologiques et/ou phytochimiques, vont être menées afin de comprendre, valider ou invalider l'usage de certaines plantes (Kamanzi, 2002; Koné, 2005; Békro et al., 2007; Zirihi et al., 2007; N'guessan et al., 2009; N'guessan et al., 2010; Adou, 2014; Mangambu et al., 2014; Lebri et al., 2015).

En Côte d'Ivoire et ailleurs dans le monde, les études phytochimiques menées sur les Ptéridophytes sont très rares, presque inexistantes. Des enquêtes ethnobotaniques réalisées ici et là ont cependant montré l'importance des Ptéridophytes comme plantes médicinales. Dans le Sud-Est de la Côte d'Ivoire, les résultats de l'enquête réalisée ont révélé que Pteridium aquilinum 
est une fougère utilisée en pharmacopée. Ce présent travail se propose donc, de valoriser Pteridium aquilinum en montrant ses différentes utilisations et en déterminant les différents groupes de composés chimiques contenus dans cette plante pour tenter d'expliquer les effets thérapeutiques potentiels de cette dernière.

\section{MATERIEL ET METHODES}

\section{Présentation de la zone d'étude}

La zone d'étude, le Sud-Est de la Côte d'Ivoire, est limitée à l'Est par le Ghana, à l'Ouest par le fleuve Bandama, au Nord par le $6^{\text {e }}$ degré de latitude Nord et au Sud par l'Océan Atlantique. Les présents travaux se sont déroulés dans les régions administratives des Lagunes, de l'Agnéby et du Sud-Comoé (Figure 1). Dans cette zone, le mois le plus pluvieux est celui de juillet et le moins pluvieux est celui de janvier. La température varie très peu et se situe autour de $26{ }^{\circ} \mathrm{C}$ en moyenne. Cette zone connaît 2 saisons. Une saison sèche (de janvier à février) et une saison pluvieuse (de mars à décembre) avec le mois d'août comme le mois de moindre pluviosité. Elle est peuplée par les Ébrié, les Attié, les Abbey, les Krobou, les Adioukrou, les Abouré, les Agni et les N'Zima. On trouve aussi des allochtones Baoulé, Malinké, etc. et des étrangers tels que les Burkinabé.

\section{Matériel biologique et technique}

Le matériel biologique étudié est Pteridium aquilinum et son extrait lyophilisé. Quant au matériel technique utilisé, il est composé entres autre d'une balance électrique, d'une hotte de protection, d'un agitateur mécanique à froid, d'un bain-marie, des capsules, des tubes, des pinces, des spatules. Les solvants utilisés pour la préparation des différents extraits sont l'éther de pétrole, l'acétate d'éthyle, le méthanol et l'eau distillée. Les réactifs utilisés, l'anhydride acétique et l'acide sulfurique $\left(\mathrm{H}_{2} \mathrm{SO}_{4}\right)$ permettent d'identifier les stérols et les polyterpènes. Les polyphénols ont été mis en évidence à partir d'une solution alcoolique de $\mathrm{FeCl}_{3}$ à $2 \%$. L'acide chlorhydrique $(\mathrm{HCl})$ dilué à $1 / 2$, les copeaux de Magnésium et l'alcool isoamylique ont été utilisés pour rechercher les flavonoïdes. La recherche des tanins a été possible grâce au réactif de Stiansny, à une solution composée de $10 \mathrm{ml}$ de formol à $30 \%$ et de $5 \mathrm{ml}$ de $\mathrm{HCl}$ concentré, à l'acétate de $\mathrm{Na}$ et à 1 goutte de solution alcoolique de $\mathrm{FeCl}_{3}$ à $2 \%$ Le réactif de Bornsträegen, l'acide chlorhydrique à $10 \%$, le chloroforme et l'ammoniac dilué à $1 / 2$ ont permis d'identifier les substances quinoniques libres ou combinées.

\section{Méthodes \\ Enquête ethnobotanique}

L'enquête ethnobotanique a permis de cerner les différentes utilisations de l'espèce par les populations. La démarche méthodologique a consisté à réaliser une enquête ethnobotanique auprès des populations du Sud-Est de la Côte d'Ivoire, précisément dans les régions administratives de l'Agnéby, des Lagunes et du Sud Comoé. Le type de méthode est une interview directe, qui consiste à interroger les populations. Les groupes sociaux ciblés sont les vendeuses de plantes médicinales, les paysans, les ménagères, les guérisseurs, les marabouts et les féticheurs. Leurs réponses sont transcrites sur une fiche d'enquête ethnobotanique (Figure 2), conçue à cet effet. Environ, 99 personnes des deux sexes et de différentes ethnies ont été interrogées.

\section{Criblage phytochimique Préparation des extraits}

Des échantillons de plante entière de Pteridium aquilinum récoltés, ont été séchés dans une enceinte climatisée, puis rendus en poudre. Quatre types d'extraits ont été obtenus à partir de la poudre de Pteridium aquilinum. Les extraits ont été obtenus après quatre phases d'extractions successives, avec des solvants à polarités croissantes. La première phase donne la solution (I) qui s'obtient selon le procédé ci-après décrit. A $20 \mathrm{~g}$ de poudre de plante dans un erlenmeyer, $200 \mathrm{ml}$ d'éther de pétrole ont été ajouté. Le tout a été ensuite 
mis sous agitation à l'aide d'un agitateur mécanique à froid pendant $30 \mathrm{mn}$ puis filtré.

Le filtrat obtenu représente la solution (I) et le marc est séché à la température du laboratoire à $18{ }^{\circ} \mathrm{C}$. Sur le marc séché, l'on ajoute successivement l'acétate d'éthyle, puis le méthanol. On obtient ainsi les solutions (II) et (III) après la filtration. Pour obtenir la solution (IV), un infusé a été préparé en ajoutant à $5 \mathrm{~g}$ de poudre végétale, $50 \mathrm{ml}$ d'eau bouillante. Ce mélange est laissé reposer pendant $15 \mathrm{mn}$ puis filtré pour obtenir l'extrait aqueux de Pteridium aquilinum.

\section{Caractérisation en tubes}

Dans ces différentes solutions, les différents composés chimiques recherchés sont les stérols, les polyterpènes, les polyphénols, les flavonoïdes et les tanins (catéchiques et galliques).

Stérols et polyterpènes: ces groupes ont été caractérisés par la Réaction de Liebermann; dans une capsule, $2 \mathrm{ml}$ d'extrait de la solution ont été évaporé. Le résidu a été dissout à chaud dans $2 \mathrm{ml}$ d'anhydride acétique. Le tout a été versé dans un tube à essai auquel avec précaution $0,5 \mathrm{ml}$ d'acide sulfurique $\left(\mathrm{H}_{2} \mathrm{SO}_{4}\right)$ a été ajouté le long de la paroi du tube. L'apparition à l'interphase d'un anneau pourpre ou violet, virant au bleu puis au vert, indique une réaction positive.

Polyphénols: la recherche est faite en utilisant le chlorure ferrique. Pour se faire, à 2 $\mathrm{ml}$ de chaque solution d'extraits, on ajoute une goutte de solution alcoolique de $\mathrm{FeCl}_{3}$ à $2 \% \mathrm{La}$ réaction positive se traduit par l'apparition d'une coloration bleu-noirâtre ou vert plus ou moins foncé (essai témoin avec une solution de phénol).

Flavonoïdes: ils ont été mis en évidence par utilisation de la réaction dite de la cyanidine. Deux (2) ml d'extrait évaporé dans une capsule. Au résidu, il a été ajouté, dans un tube à essais, $5 \mathrm{ml}$ de $\mathrm{HCl}$ dilué à demi, 3 copeaux de Mg et la coloration est notée. Puis le long de la paroi du tube, $0,5 \mathrm{ml}$ d'alcool isoamylique ont été versé. Ce dernier se colore en rose, rouge ou rouge orangé en présence des flavonoïdes. Le témoin est une solution alcoolique de quercétine.

Tanins: A partir du réactif de Stiansny, ces composés ont été recherchés en faisant évaporer $5 \mathrm{ml}$ de chaque solution d'extrait. Ensuite $15 \mathrm{ml}$ d'une solution composée de 10 $\mathrm{ml}$ de formol à $30 \%$ et de $5 \mathrm{ml}$ de $\mathrm{HCl}$ concentré ont été ajouté au résidu. Le mélange a été maintenu au bain-marie à $80^{\circ} \mathrm{C}$ pendant $30 \mathrm{mn}$ après lesquelles il est laissé refroidir. De gros flocons brun-clair se forment en présence de tanins catéchiques. Cette solution est ensuite filtrée et saturer d'acétate de $\mathrm{Na}$. Une (1) goutte de solution alcoolique de $\mathrm{FeCl}_{3}$ à $2 \%$ y est additionnée. La réaction positive se traduit par l'apparition d'une coloration bleunoir intense qui caractérise les tanins galliques, non précipités par le réactif de Stiansny. Le témoin utilisé est une solution alcoolique d'acide gallique et de la catéchine.

Quinones: les substances quinoniques libres ou combinées ont été mises en évidence à partir du réactif de Bornsträegen. Deux (2) $\mathrm{ml}$ de chacun des extraits a été évaporé à sec dans une capsule. $\mathrm{Au}$ résidu, l'acide chlorhydrique à $10 \%$ a été ajouté. La solution est ensuite portée au bain-marie bouillant pendant $30 \mathrm{mn}$. Après refroidissement, l'hydrolysat a été épuisé par $20 \mathrm{ml}$ de $\mathrm{CHCl}_{3}$, puis il a été ajouté $0,5 \mathrm{ml}$ d'ammoniac dilué au démi. Une coloration rose ou rouge indique la présence de quinones. Le témoin utilisé est une solution chloroformique d'anthraquinone.

\section{RESULTATS \\ Etude ethnobotanique}

Quelques informations sur Pteridium aquilinum ont pu être obtenues auprès des populations. Sur les 99 personnes interrogées, 62 connaissent la plante et 37 ne la connaisse pas. 33 n'en savent pas l'utilité et 29 soit $29 \%$ de personnes enquêtées connaissent la plante et en savent l'utilité. Les résultats concernant la plante recensée et ses indications thérapeutiques sont consignés dans le Tableau 1. 


\section{Organes utilisés, modes de préparation et d'administration des médicaments}

Plusieurs parties de la plante entrent dans la préparation de ces médicaments. Toutefois, la plante entière est utilisée dans $66 \%$ des cas, ensuite viennent les frondes fraîches avec $33 \%$. Le pétrissage et la décoction sont les recettes (modes de préparation) des remèdes selon les enquêtés. La décoction est le mode de préparation le plus sollicité avec $66 \%$. Le décocté et la pâte sont donc les formes médicamenteuses. Les médicaments obtenus ici à base de Pteridium aquilinum sont administrés sous forme de boisson $(33 \%)$, de bain de vapeur $(33 \%)$ et d'onguent (33\%).

\section{Analyse phytochimique qualitative}

Le Tableau 2 donne les résultats de la caractérisation en tube des composés chimiques sur les 4 extraits de Pteridium aquilinum.

L'absence de tanins galliques a été notée dans tous les quatre extraits. C'est aussi le cas des polyphénols qui sont absents dans les extraits de l'éther de pétrole. Les flavonoïdes sont présents uniquement dans l'extrait aqueux. Les tanins catéchiques et les substances quinoniques, sont absents dans les extraits à l'éther de pétrole. Les stérols et les polyterpènes sont présents dans les extraits à l'acétate d'éthyle et à l'éther de pétrole.

Tableau 1 : Indications thérapeutiques, recettes et modes d'administration de Pteridium aquilinum.

\begin{tabular}{llllll}
\hline $\begin{array}{l}\text { Nom } \\
\text { d'espèce }\end{array}$ & $\begin{array}{l}\text { Indications } \\
\text { thérapeutiques }\end{array}$ & $\begin{array}{l}\text { Organes } \\
\text { utilisés }\end{array}$ & Recettes & $\begin{array}{l}\text { Formes } \\
\text { médicamenteuses }\end{array}$ & Modes d'administration \\
\hline & & $\begin{array}{l}\text { Plante } \\
\text { entière }\end{array}$ & $\begin{array}{l}\text { décoction } \\
\text { de la plante } \\
\text { entière. }\end{array}$ & Décocté & $\begin{array}{l}\text { Bain de vapeur } \\
\text { sur l'articulation malade }\end{array}$ \\
$\begin{array}{l}\text { Pteridium } \\
\text { aquilinum }\end{array}$ & + & Rhumatisme & Frondes & Pâte & $\begin{array}{l}\text { Onguent sur l'articulation } \\
\text { malade }\end{array}$ \\
& & fraîches & $\begin{array}{l}\text { pétrissage des } \\
\text { frondes (feuilles) } \\
\text { fraîches }\end{array}$ & & \\
& & & $\begin{array}{l}\text { La décoction de } \\
\text { la plante entière }\end{array}$ & Décocté & Boisson \\
\cline { 2 - 6 } & Convulsions des & $\begin{array}{l}\text { Plante } \\
\text { entière }\end{array}$ & &
\end{tabular}

Tableau 2 : Résultat synthétique de la caractérisation en tubes des composés chimiques.

\begin{tabular}{|c|c|c|c|c|c|c|}
\hline Extraits & $\begin{array}{l}\text { Stérols et } \\
\text { polyterpènes }\end{array}$ & Polyphénols & Flavonoïdes & $\begin{array}{l}\text { Tanins } \\
\text { galliques }\end{array}$ & $\begin{array}{l}\text { Tanins } \\
\text { catéchiques }\end{array}$ & $\begin{array}{l}\text { Substances } \\
\text { quinoniques }\end{array}$ \\
\hline PA SI & + & - & - & - & - & - \\
\hline PA SII & + & + & - & - & + & + \\
\hline PA SIII & - & + & - & - & + & + \\
\hline PA SIV & - & + & + & - & + & + \\
\hline $\begin{array}{l}\text { PA S } \\
\text { PA S } \\
\text { PA S } \\
\text { PA S } \\
+=\mathrm{I} \\
-=\mathrm{ab}\end{array}$ & $\begin{array}{l}\text { Solution de Pterid } \\
=\text { Solution de Pteri } \\
=\text { Solution de Pter } \\
=\text { Solution de Pter } \\
\text { sence } \\
\text { nce }\end{array}$ & $\begin{array}{l}\text { m aquilinum } \mathrm{pré} \\
\text { um aquilinum } \mathrm{pr} \\
\text { ium aquilinum } \mathrm{p} \\
\text { ium aquilinum } \mathrm{p}\end{array}$ & $\begin{array}{l}\text { ee avec l'éther d } \\
\text { ée avec l'acétat } \\
\text { rée avec le méth } \\
\text { rée avec l'eau }\end{array}$ & $\begin{array}{l}\text { étrole } \\
\text { 'éthyle } \\
\text { ol }\end{array}$ & & \\
\hline
\end{tabular}




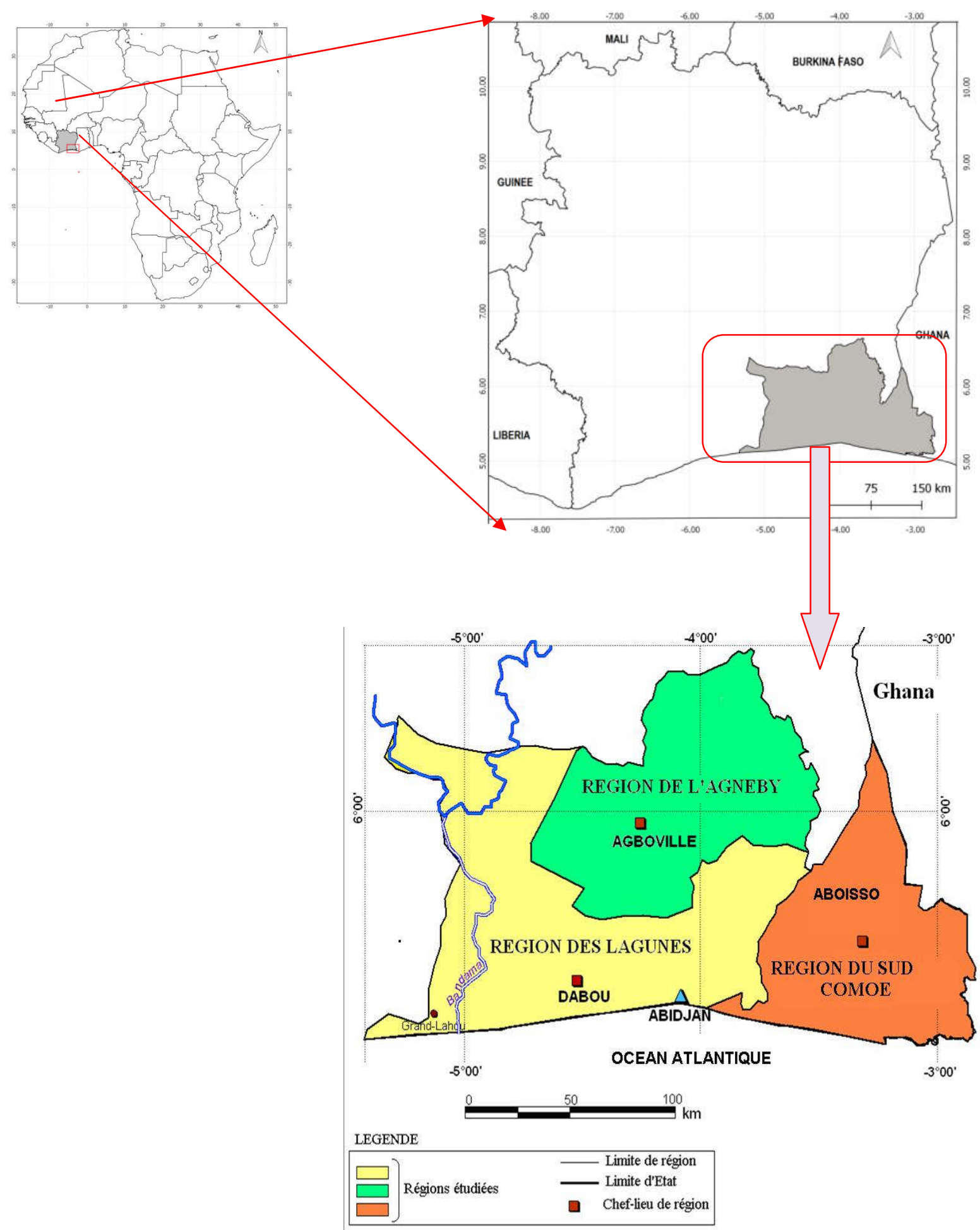

Figure 1: Localisation de la zone d'étude (Centre National de Télédétection et d'Information Géographique (C.N.T.I.G), 2001). 
$\mathrm{N}^{\circ}: 27$

Localité : Dabou

Enquêteur : Date : 9/11/1999

Sexe : Masculin

Ethnie : Adioukrou

Profession ou activité : Agriculteur

Enfant : Jeune

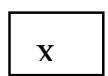
Adulte

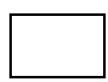

Vieux

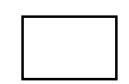

Nom scientifique de la plante: Pteridium aquilinum

Questions :

Connaissez-vous la plante?

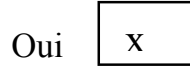

Non

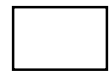

Par qui la connaissez-vous? Mes parents

Connaissez-vous des utilisations de cette plante?

Si oui, lesquelles?

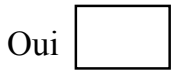

Non

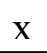

Quelle partie de la plante est-elle utilisée?

Et les autres parties?

Quel est le nom de la plante dans votre ethnie ?ne connaît pas le nom vernaculaire

Figure 2 : Fiche d'enquête ethnobotanique

\section{DISCUSSION}

Pteridium aquilinum est une plante importante aussi bien du point de vue de la diversité biologique que pour les besoins de l'Homme. Dans le Sud-est de la Côte d'Ivoire, Cette plante est diversement utilisée par les populations qui s'en servent généralement pour guérir certaines maladies. En effet, elle rentre dans la composition de plusieurs médicaments. D'autres études mentionnent également l'utilisation de cette espèce dans la médecine traditionnelle. Mangambu et al. (2012), Latham et Konda (2007); Konda et al. (2011) signalent que Pteridium aquilinum est utilisée dans le traitement des douleurs thoraciques et des hémorroïdes. Pour Mangambu et al. (2012), en République démocratique du Congo, la macération d'une poignée de frondes fraîches de cette même plante dans 2 litres d'eau, donne après 12 heures, un liquide qui est utilisé par voie rectale pour traiter le diabète. En Europe également, Bonet et al. (2001), soutiennent que la décoction du rhizome de cette même espèce est utilisée comme diurétique dans les Pyrénées. Si la plante entière est sollicitée à $66 \%$ pour la préparation des médicaments dans cette étude, ce résultat n'est pas conforme à ceux de N'guessan et al. (2009) et de Mangambu et al. (2014). Ils ont montré respectivement que chez les Krobou en Côte d'Ivoire et en République Démocratique du Congo, précisément dans la ville de Bukavu et ses environs, c'étaient les feuilles qui étaient majoritairement sollicitées dans $63,52 \%$ des cas et majoritairement employées dans $63,63 \%$ des cas. La décoction (66\%) est le mode de préparation le plus sollicité dans cette étude. Ces résultats concordent avec ceux des travaux de Ouattara (2006) et N'guessan et al. (2009) qui indiquent que la décoction est sollicitée respectivement dans $43,75 \%$ des cas, dans le Département de Divo et dans $42,30 \%$ dans le Département d'Agboville. C'est le mode de préparation le plus courant dans les traitements par les plantes pour la grande majorité des tradithérapeutes.

Les médicaments obtenus ici à base de Pteridium aquilinum sont administrés sous forme de boisson, de bain de vapeur et d'onguent. Chacun de ces trois modes est utilisé dans $33 \%$ des cas. D'autres études menées par certains auteurs tel que Ouattara (2006); N'guessan et al. (2009); Yapi et al. (2015) indiquent que seul le mode d'administration sous forme de boisson était 
le plus sollicité avec respectivement $32,35 \%$, $48,97 \%$ et $35,94 \%$.

Les résultats de l'enquête ont montré dans ce travail que des recettes médicamenteuses à base de Pteridium aquilinum se proposent de guérir plusieurs maux que sont entre autres les convulsions des bébés et le rhumatisme. Ces résultats sur le rhumatisme et ceux de Latham et Konda (2007); Konda et al. (2011) sur les douleurs thoraciques laissent penser que cette plante pourrait avoir des propriétés antalgiques, pour la simple raison que ces deux indications thérapeutiques (rhumatisme et douleurs thoraciques) sont manifestées par des douleurs. Quant à la guérison des convulsions des bébés par cette plante, l'on peut penser également que l'espèce pourrait avoir des propriétés antipyrétiques. En effet, cette maladie qui attaque les nouveaux nés se manifeste généralement par des contractions musculaires liées à une forte fièvre. Les effets thérapeutiques de Pteridium aquilinum sont dus à la présence de certaines substances. La probable guérison du rhumatisme par cette plante serait le fait des flavonoïdes dont les propriétés anti-inflammatoires sont signalées par Benguerba (2008) in Bouziane (2014). Le screnning phytochimique seul n'étant pas suffisant pour justifier l'utilisation d'une plante en médecine traditionnelle, nous envisageons de compléter cette étude en évaluant la toxicité de cette plante pour l'Homme.

\section{Conclusion}

Cette étude a montré que Pteridium aquilinum est utilisée pour traiter le rhumatisme et les convulsions des bébés. Cette espèce renferme plusieurs groupes de composés chimiques que sont les stérols, les polyterpènes, les polyphénols, les flavonoïdes, des tanins catéchiques et des substances quinoniques. La présence de ces composés constitue une base scientifique de l'utilisation thérapeutique traditionnelle de la plante étudiée. Les effets thérapeutiques sont induits par ces divers composés chimiques. Il est donc indispensable de connaitre la composition des plantes pour comprendre comment elles agissent potentiellement sur l'organisme. Cette étude, pourra être complétée en évaluant la toxicité de cette plante pour l'Homme, sans toutefois oublier de réaliser encore des enquêtes ethnobotaniques sur l'espèce dans d'autres régions; en effet les différents composés chimiques déterminés indiquent que cette plante pourrait exercer divers effets thérapeutiques que les enquêtes dans le SudEst de la Côte d'Ivoire ne signalent pas.

\section{CONFLIT D'INTERETS}

Les auteurs déclarent qu'ils n'ont aucun conflit d'intérêts.

\section{CONTRIBUTIONS DES AUTEURS}

LMDA a réalisé les enquêtes ethnobotaniques et a contribué à la rédaction du manuscrit; MWK, a contribué à l'analyse phytochimique qualitative de la plante; JII a contribué à la réalisation de la fiche d'enquête et à la rédaction du Manuscrit; EKN a contribué par sa lecture à l'amélioration du manuscrit.

\section{REMERCIEMENTS}

Nous remercions le Centre National de Floristique de Côte d'Ivoire pour avoir servi de cadre à la détermination de l'espèce. , Ainsi que le Centre Suisse de Recherches Scientifiques (CSRS) Côte d'Ivoire pour avoir servi de cadre pour l'analyse Phytochimique

\section{REFERENCES}

Adou LMD, Touré A, Komoé K, Ipou Ipou J. 2014. Nephrolepis biserrata, une Ptéridophyte utilisée comme plante médicinale en Côte d'Ivoire. Journal of Applied Biosciences, 81:7298-7306.

Azokou A. 2010. Activité Biolarvicide de quelques espèces végétales de côte d'Ivoire contre les moustiques Culex quiquefasciatus et Anopheles gambiae, vecteurs de maladies. DEA de G.V.R.N., UFR-SN, Université Abobo-Adjamé Abidjan-Côte d'Ivoire, p73. 
Baldé NM, Diallo L, Baldé MD, Barry LS, Kaba L, Diallo MM, Kaké A, Camara A, Bah D Barry MM, Bah-Sangaré M, Maugendre D. 2006. Diabetes and impaired fasting glucose in rural and urban populations in Futa Jallon (Guinea): prevalence and associated risk factors. Diabetes \& Metabolism (in press)

Békro YA, Békro JAM, Boua BB, Tra Bi FH, Ehilé E. 2007. Étude ethnobotanique et screening. phytochimique de Caesalpinia benthamiana (Baill.) Herend. et Zarucchi (Caesalpiniaceae). Sciences \& Nature, 4(2) : $217-225$.

Benguerba A. 2008. Etude Phytochimique et de la Phase Butanolique de L'espèce Inula crithmoides L. Thèse de Magister En Chimie Organique Option Phytochimie, Universite mentouri constantine, Algerie.

Bonet MA. Agelet A, Vallès J, Villar L. 2001. Contribution à la connaissance ethnobotanique des ptéridophytes dans les Pyrénées. Bocconea, 13: 605-612.

Bouziane C. 2014 Etude de l'interaction de la phospholipase A2 et flavanones isolées de l'Inule Visqueuse par modélisation moléculaire: Mémoire de Master, Université abou Bekr Belkaid Tlemcen, Algerie, p. 46.

Bruneton J. 2009. Pharmacognosie: phytochimie, plantes médicinales (4th ed., pp. 567-961. Paris: Technique et Documentation.

Centre National de Télédétection et d'Information Géographique (C.N.T.I.G). 2001. Carte Administrative de la République de Côte d'Ivoire, Edition CEDA, $1 p$.

Dossou ME, Houessou GL, Lougbégnon, OT, Tenté AHB, Codjia JTC. 2012. Etude ethnobotanique des ressources forestières ligneuses de la forêt marécageuse d'Agonvè et terroirs connexes au Bénin. Rev.Tropicultura, 30(1): 41-48.

Gning SB, Thiam M, Fall F, Ba-Fall K, Mbaye PS, Foucarde L. 2007. Le diabète sucré en Afrique subsaharienne: aspects épidémiologiques, difficultés de prise en charge. Médecine Tropicale, 67: 607-611.

Kamanzi A. 2002. Plantes médicinales de Côte-d'Ivoire : investigations phytochimiques guidées par des essais biologiques. Thèse de Doctorat d'Etat, Université de Cocody-Abidjan, UFR Biosciences, $\mathrm{N}^{\circ}$ d'ordre: 363/2002, $176 \mathrm{p}$.

Konda K, Kabukura M, Mbembe B, ItufaY, Mahuku K, Mafuta M, Mpoyi K, Ndemankeni I, Kadima K, Kélela B, Ngiuvu V, Bongombola M, Dumu L. 2011. Plantes médicinales de traductions de la province de l'Equateur en RD CONGO).Institut de recherche en sciences de la santé (Kinshasa), 418 p.

Koné MW. 2005. Potentiel des plantes médicinales de Côte-d'Ivoire dans le contrôle des haemonchoses chez les ovins. Thèse de Doctorat de l'Université de Cocody, UFR Biosciences, Laboratoire de Botanique, $202 \mathrm{p}$.

Latham P, Konda K. 2007. Plantes Utiles $d u$ Bas-Congo, République Démocratique du Congo (2ème édition). DFID: UK ; $330 \mathrm{p}$. Lebri M, Bahi C, Fofié NBY, Gnahoue G, Lagou SM, Achibat H, Yapi A, Zirihi GN, Coulibaly A, Hafid A, Khouili M. 2015. Analyse phytochimique et évaluation de la toxicité aiguë par voie orale chez des rats de l'extrait total aqueux des feuilles de Abrus precatorius Linn. (Fabaceae). Int. J. Biol. Chem. Sci., 9(3): 1470-1476.

Mangambu JD, Van Diggelen R, Mwanga Mwanga JC, Ntahobavuka H, Malaisse F, Robbrecht E. 2012. Check-list des Ptéridophytesde l'écosystème forestier des montagnes du Parc National de Kahuzi-Biega à l'Est de la R.D Congo. Cahier du Centre de Recherches Universitaires $d u K i v u$, nouvelle série, 42(2): 363-374.

Mangambu JD, Mushagalusa KF, Kadima NJ. 2014. contribution à l'étude phytochimique de quelques plantes médicinales antidiabétiques de la ville de Bukavu et ses environs (Sud-Kivu, 
R.D.Congo). Journal of Applied Biosciences, 75: 6211-6220.

N'guessan K, Kadja B, Zirihi GN, Traoré D, Aké-Assi L. 2009. Screening phytochimique de quelques plantes médicinales ivoiriennes utilisées en pays krobou (Agboville, Côte d'Ivoire). Rev. Sciences \& Nature, 06(1): 1 -15.

N'Guessan K, Zirihi NG, Boraud N. 2010. Etude ethnopharmacologique des plantes utilisées pour faciliter l'accouchement, en pays Abbey et Krobou, au Sud de la Côte d'Ivoire. International Journal of Biological and Chemical Sciences, 4(4): 1004-1016.

Ouattara D. 2006. Contribution à l'inventaire des plantes médicinales significatives utilisées dans la région de Divo (sud forestier de la Côte d'Ivoire) et à la diagnose du poivrier de guinée: Xylopia aethiopica (Dunal) A. Rich. (Annonaceae). Thèse de Doctorat de l'Université de Cocody-Abidjan (Côte d'Ivoire), UFR Biosciences, Laboratoire de Botanique, $184 \mathrm{p}$.

Sanogo R, Diallo D, Diarra S, Ekoumou C, .Bougoudogo F. 2006. Activité antibactérienne et antalgique de deux recettes traditionnelles utilisées dans le traitement des infections urinaires et la cystite au Mali. Rev. Mali Médical T XX(1): 18-24.
Singh B, Singh BK. 2012. Ethnomedicinal use of Pteridophytes in reproductive health of tribal women of Pachmarhi Biosphere Reserve, Madhya Pradesh, India. International Journal of Medicine and Medical researcher, 3(12): 4780-4790.

Tra Bi FH, Irié GM, Kohué CC, N'Gaman, Clejesson HB, Mohou. 2008. Etudes de quelques plantes thérapeutiques utilisées dans le traitement de l'hypertension artérielle et $\mathrm{du}$ diabète: deux maladies émergentes en Côte d'Ivoire. Sciences \& Nature, 5(1): 39-48.

Yapi AB, Kassi NJ, Fofié NBY, Zirihi GN. 2015. Etude ethnobotanique des Astéraceae médicinales vendues sur les marchés du district autonome d'Abidjan (Côte d'Ivoire). Int. J. Biol. Chem. Sci., 9(6): 2633-2647.

Zirihi GN., Datté JY, Kra-Adou KM. Grellier P. 2007. Phytochemical and pharmacological studies of the alcoholic extract (MFA) of Fagara macrophylla (Oliv.) Engl. (Rutaceae): the chemical structure of the active compoundin ducing antipaludic activity. Journal of Chinese Clinical Medicine, 2(4): 205-21. 